\title{
Heroic holograms
}

\section{J. Bishop}

ONE of the pre-eminent intellectual challenges in condensed matter physics is understanding the behaviour of magnetic vortices in the oxide superconductors at the microscopic level ${ }^{1-4}$. In part, this is because the vortices are a nearly ideal model system in which to study the competing effects of disorder, correlation, anisotropy, thermal fluctuations and dimensionality. Another component is the unrequited desire to find commercial applications for these materials, especially in uses that require large critical currents. In the past several years, both experimental ${ }^{5-10}$ and theoretical ${ }^{3}$ techniques of unprecedented difficulty and sophistication have been brought to bear on the problem. It is a golden era for research in this field.

In 1992, workers at Hitachi developed a technique for imaging magnetic flux lines in a superconductor using electron holography ${ }^{10}$, which allowed them to see the behaviour of the lines in real time, as a function of magnetic field, temperature and applied current. In the initial work, they studied niobium; at the time, it was not clear that they would ever be able to apply the technique to the technologically more important but much more difficult high- $T_{\mathrm{c}}$ superconductors. But in this week's issue of Physical Review Letters, Harada and colleagues ${ }^{5}$ describe

how they have successfully adapted their method to image magnetic vortices in an oxide superconductor, $\mathrm{Bi}: 2212$ (formula $\left.\mathrm{Bi}_{2+x} \mathrm{Sr}_{2-x} \mathrm{CaCu}_{2} \mathrm{O}_{8+\delta}\right)$. It is a truly outstanding piece of experimental physics which moves the bar up another notch for state-of-the-art experiments in this field.

All of the recently discovered oxide superconductors are in the 'type II' class. For these materials, the distance over which magnetic fields can decay (the penetration depth) is much larger than the distance over which the electronic properties such as the superconducting energy gap can change (the coherence length). As was first shown by Abrikosov in the 1950s, conventional type II superconductors can do one of three things when a magnetic field is applied. At low fields and temperatures, they can expel the field (the Meissner state). At high fields and/or temperatures, the field can destroy the superconducting state producing a normal metal. However at intermediate fields (the mixed state), the magnetic field enters the
False-colour image of a magnetic flux-line lattice in a crystal of $\mathrm{Bi}: 2212$, as seen using magnetic decoration. The flux lines are the red dots and are roughly 1 micrometre apart in the applied field of 20 gauss. Note that the vortices form a triangular lattice. superconductor in the form of quantized bundles of magnetic flux called magnetic flux lines. In the absence of disorder, these flux lines will form a well-ordered triangular flux lattice.

In the oxide superconductors, experiments have indicated that still another phase can exist - a liquid of flux lines and that the flux lattice can melt into this flux-line liquid. We care about magnetic

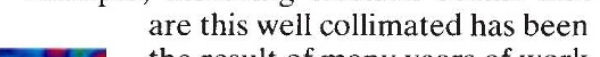
are this well collimated has been the result of many years of work at Hitachi's Advanced Research Laboratory. To do these heroic experiments, which would be impossible in a conventional laboratory environment, they have been forced to build a separate building in which they are isolated from all sources of mechanical, electrical and magnetic noise. These workers will have very little competition.

In this week's paper, Harada and co-workers ${ }^{5}$ report that they have observed vortices in a film of $\mathrm{Bi}: 2212$ and have been able to follow them up through the melting temperature of the flux lattice. At low temperatures, they observe a roughly triangular lattice of vortices. As they raise the temperature, they see that at each temperature step the vortices move a little, but eventually settle into a new static configuration. However, at the irreversibility temperature (but well below $T_{c}$ ) they see the lattice fade away. Thus they are the first to flux lines because a flowing electric current in a superconductor induces a force on them. If they can respond to this force and are free to move, they will dissipate energy in the flowing current and produce an effective resistance. This is especially true in the vortex liquid state. In this case, these superconductors aren't even good conductors.

It is clear that imaging techniques will be of key importance in unravelling the dynamics of such magnetic flux-line lattices. Those developed over the years include magnetic decoration ${ }^{6}$, STM imaging ${ }^{7}$, small-angle neutron scattering ${ }^{8}$, scanned Hall bars ${ }^{9}$ and the new entrant in the field, electron holography ${ }^{10}$. For example, shown in the figure is an image of the flux-line lattice in a crystal of $\mathrm{Bi}: 2212$ as seen using magnetic decoration. One can clearly see the individual flux lines and the triangular lattice that they form. But the problem with this technique and most of the others is that they do not allow one to image the lattice and follow its dyna- generate real-space, real-time images of a melting magnetic flux-line lattice in a type II superconductor. It is an experimental tour de force.

Many of us have been consumed for the past six years by the issue of whether magnetic flux lattices could indeed melt. We have argued for quite some time that they do melt, but seeing is believing - to those in the superconductivity business, these images are nothing less than spectacular.

David Bishop is at AT\&T Bell Laboratories, Murray Hill, New Jersey 70974, USA.

\footnotetext{
1. Bishop, D. J. et al. Scient. Am. 268, 48-55 (1993).

2. Bishop, D. J. et al. Science 255, 165-172 (1992).

3. Huse, D. A. et al. Nature 358, 553-559 (1992).

3. Huse, D. A. et al. Nature 358, 553-559 (1992
4. Bishop, D. J. Nature 365, 394-395 (1993).

4. Bishop, D. J. Nature 365, 394-395 (1993).

5. Harada, K. et al. Phys. Rev. Lett. 71, 3371 (1993).
6. Gammel, P. L. et al. Phys. Rev. Lett. 59, 2592-2595 (1987)

7. Hess, H. et. al. Phys. Rev. Lett. 62, 214-217 (1989).

8. Cubitt, R. et. al. Nature 365, 407--411 (1993).

9. Hallen, H. D. et al. Phys. Rev. Lett. 71, 3007-3010 (1993).

10. Harada, K. et al. Nature 360, 51-53 (1993).
} 